\title{
THE HEART RATE DURING A SIMPLE EXERCISE
}

\author{
BY \\ J. A. C. KNOX \\ From the Department of Physiology, University of Glasgow \\ Received July 12, 1940
}

Simple exercise tolerance tests, such as stepping on and off a stool a given number of times and noting the effect on the pulse rate, have long been used in medical practice. Almost invariably, however, the pulse rate is taken before and after, owing to the difficulty of counting it during the exercise. This may be overcome by means of a recording apparatus such as that of Bell and Knox (1938); the R-S waves of the electrocardiogram are recorded on a smoked drum, and the amplifier is modified to minimize the effects of skeletal muscle activity. Time in seconds, respiration, and signals for the beginning and end of exercise are also recorded on the drum, and thus a very complete and accurate analysis of the heart rate during even a short exercise is possible. Fig. 1 shows a normal trace obtained by this method.

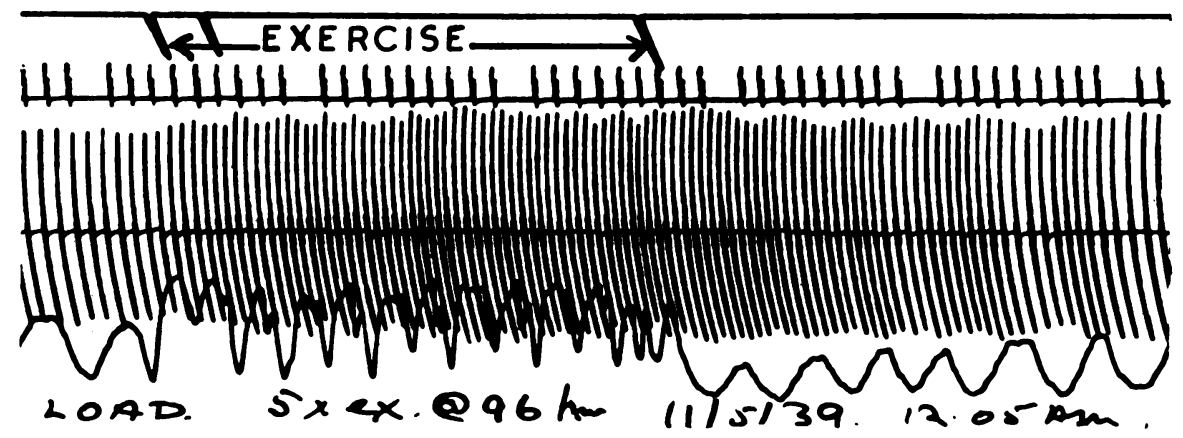

Fig. 1.-Portion of normal tracing showing the response to exercise. Upper tracing, signal showing the start and stop of the exercise. Second tracing, time in seconds, with a break once in 10 seconds. Third tracing, heart beats. Fourth tracing, respiration (the rise of the lever indicates expiration).

The exercise adopted as standard for this series is light enough to be suitable for many cardiac patients, and the movements required, viz. those of stairclimbing, enter into the routine of daily life. The advantage of this was pointed out by Master and Oppenheimer (1929) and by White (1937).

Seventy-five male medical students acted as subjects. They were in good health, but were not in training. Their ages, in nearly every case, lay between 18 and 22 years. 


\section{The Tolerance Test}

As the test is designed with a view to clinical use, no extreme precautions are taken for obtaining a true basal initial rate, and slight latitude is allowed in the performance of the exercise. The subject first has a brief practice in ascending the steps, and after the chest electrodes and stethograph have been applied, he sits down on a chair eighteen inches high and relaxes as fully as possible.

The two chest electrodes employed are saucer-shaped copper discs $2.5 \mathrm{~cm}$. in diameter. The concavity is filled with soft green soap which acts as the electrolyte, as recommended by Bell, Knox, and Small (1939). The electrodes are kept in contact with the chest by means of light elastic straps. One electrode is applied over the apex beat and the other over the second right costal cartilage. A satisfactory voltage and an even base line are obtained with the electrodes in these positions.

At the end of three minutes a tracing is run for 30 seconds, the subject remaining relaxed. The drum is then stopped and a further two minutes' rest allowed, giving five minutes in all; Master (1934) stated that this is usually sufficient to reach a steady resting rate. The drum is then started and the resting heart rate recorded for 15-20 seconds, after which the signal to start the exercise is given. The subject rises to his feet, steps five times up and down two steps each ten inches high and then sits down again and relaxes. To standardize the rate of climb the subject steps in time with a metronome beating 96 per minute. The average duration of the exercise from rising to sitting again is 24 seconds. It will thus be seen that the standard exercise of the present test is both lighter and of much shorter duration than that of the usual type of cardiac tolerance test.

\section{Method of Analysing the Tracings}

Fine vertical lines are drawn across the tracings at intervals of 5 seconds, starting 10 seconds before the beginning of the exercise. The heart rate is then counted (to the nearest tenth of a beat) in each of these intervals of 5 seconds, and the highest rate obtained is converted to beats per minute; this is taken as the maximum rate. The time to reach the maximum rate is taken from the beginning of exercise to the middle of the 5-seconds period with the maximum rate. For example, if the maximum rate is found in the third 5 seconds from the beginning of the exercise the time to reach the maximum rate is 12.5 seconds.

The heart rate in the 10 seconds immediately preceding the beginning of the exercise, converted to beats per minute, is taken as the initial rate.

The actual increase in beats per minute of the maximum rate over the initial rate is given, and this is also expressed as a percentage of the initial rate (percentage increase on initial rate).

The acceleration of the heart rate in beats-per-minute per second is calculated by subtracting the rate in the 5 seconds immediately preceding exercise (converted to beats per minute) from the maximum rate, and dividing the resulı by the time taken to reach the maximum rate.

Another index which may prove of interest is the number of extra heart 
beats induced by the exercise. This is calculated by subtracting half the initial rate (in beats per minute) from the number of beats in the 30 seconds after starting the exercise, this including the whole of the exercise period.

To get an index that does not require the use of apparatus, the heart rate is counted over the 30 seconds beginning 5 seconds after exercise ends, this being called the post-exercise rate and expressed in beats per minute.

\section{Example of the Analysis of a Typical Tracing}

The heart beats in the successive 5 seconds, starting 10 seconds before the beginning of exercise, were as follows: $6 \cdot 9,7 \cdot 0,8 \cdot 2,10 \cdot 0,10 \cdot 7,10 \cdot 5,9 \cdot 1,8 \cdot 6$, 8.4. The duration of exercise was 25 seconds. The initial rate is therefore $(6.9+7 \cdot 0) \times 6=83.4$ beats per minute.

The maximum rate is $10.7 \times 12=128.4$ beats per minute, and it occurs in the third 5 seconds from the beginning of exercise; therefore the time to reach the maximum rate is taken as 12.5 seconds.

The acceleration of the heart rate is $(128.4-83.4) \div 12 \cdot 5=3.60$ beats-perminute per second.

The number of beats in the 30 seconds after exercise began was $57 . \quad$ Thus the number of extra beats induced by the exercise was $57-1 / 2(83 \cdot 4)=15 \cdot 3$ beats.

The percentage increase on the initial rate was $(128 \cdot 4-83 \cdot 4) \times 100 \div 83 \cdot 4=54$ per cent.

The actual increase in beats per minute was $128 \cdot 4-83 \cdot 4=45$ beats per minute.

The results are shown in Table $I$.

TABLE I

The Rise of Pulse Rate with this Exercise Tolerance Test in 75 Healthy Male Students

\begin{tabular}{|c|c|c|c|c|c|}
\hline Index & & Mean & Range & $\begin{array}{l}\text { Standard } \\
\text { Deviation }\end{array}$ & $\begin{array}{c}\text { Coefficient } \\
\text { of } \\
\text { Variation, } \\
\text { Percentage }\end{array}$ \\
\hline 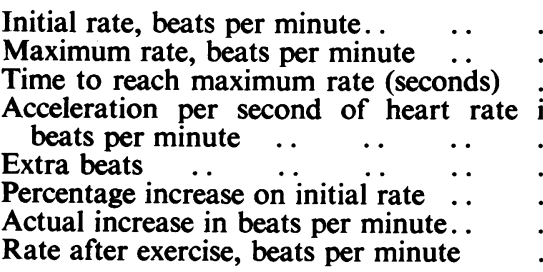 & $\begin{array}{l}\text {. } \\
\cdots \\
\text { in } \\
\cdots \\
\cdots \\
\cdots \\
\cdots\end{array}$ & $\begin{array}{r}85 \cdot 7 \\
130 \cdot 2 \\
15 \cdot 0 \\
\\
3 \cdot 0 \\
17 \cdot 0 \\
53 \cdot 9 \\
44 \cdot 4 \\
91 \cdot 7\end{array}$ & $\begin{array}{c}59-121 \\
109-168 \\
7 \cdot 5-22 \cdot 5 \\
\\
1 \cdot 4-5 \cdot 3 \\
8 \cdot 6-27 \cdot 2 \\
19 \cdot 8-100 \\
25-69 \\
60-134\end{array}$ & $\begin{array}{r}0.9 \\
4 \cdot 9 \\
18 \cdot 5 \\
10 \cdot 1 \\
14 \cdot 8\end{array}$ & $\begin{array}{l}28 \cdot 7 \\
28 \cdot 6 \\
34 \cdot 3 \\
22 \cdot 8 \\
16 \cdot 2\end{array}$ \\
\hline
\end{tabular}

Discussion OF RESULTS

(1) Initial Rate.-The mean rate of 85.7 beats per minute is somewhat higher than might be expected. The average sitting pulse rate in a corresponding age group was found to be 71-72 per minute by Volkmann (1850), and 70-72 per minute by McCurdy and Larson (1939), though Langowoy 
(1900) gave an average of 80 per minute in a small series. The high initial rate of the present series is probably the result of psychological factors, as the subjects know that the exercise is about to start, though care is taken to see that the apparatus is not visible to them. This is borne out by the fact that the average heart rate taken after the first three minutes of rest is 81.5 per minute, while after a further two minutes of rest it has risen to 85.7 per minute just before exercise. Peabody and Sturgis (1922) found the same effect in normal subjects and in cardiac patients, and Addis (1922) showed the effect of excitement on the resting pulse rate. The accelerating action of mental factors on the heart was also pointed out by Gillespie (1924), Grollman (1929), and Ellis (1932).

Data on the variability of the sitting pulse rate appear to be very scanty, but Addis (1922) gave the average recumbent basal pulse rate as 80 per minute, and Jackson (1927) found an average recumbent rate of 80.2 per minute in 1600 male students, the coefficients of variation being 16 and $15 \cdot 1$ per cent respectively. Thus the coefficient of variation of the sitting pulse rate in the present series agrees well with these results quoted.

(2) Maximum Rate.-It is surprising that the mean maximum rate in a 5 -seconds interval should be as high as 130 per minute in healthy young men performing a very light exercise. The maximum rate would appear to be a suitable index for measuring cardiac tolerance, as it is the least variable of the indices chosen, its coefficient of variation being only 9.9 per cent. If the initial heart rate is high the maximum rate reached is also high, the coefficient of correlation being +0.69 with a standard error of $0 \cdot 116$. This does not mean, however, that emotion simply raises the levels of the heart rates evenly throughout the experiment. In fact, where the initial rate is high the increase in the number of beats per minute produced by the exercise is generally low. For example, if the subjects are divided into those with initial rates below and above 85 per minute, the increase with exercise averages 48 beats per minute in the former, and $\mathbf{4 0}$ beats per minute in the latter.

This smaller increase is particularly noticeable in those whose resting rate just before exercise shows much increase over the resting rate two minutes earlier; these are presumably the subjects in whom the emotional element is greatest. It would thus appear that during the exercise the emotional increase is lessened and that some factor comes into play to prevent an excessively high maximum rate. Cotton, Rapport, and Lewis (1917) found a similar relationship between the initial and maximum rates recorded after the end of maximal exercise. They explained it on the assumption that " the circulation is capable at a given moment of a certain response to a given effort, and it appears to be a matter of indifference whether this response has been called forth to. some extent before the chief stimulation has been applied, providing it has been called forth through similar channels." Boas and Goldschmidt (1932) made similar observations. In the present series, if emotion is considered to play the part of the introductory stimulus before the chief stimulus is applied, the results are in accordance with this theory, although the exercise is very far short of maximal. 
(3) Time of Occurrence of the Maximum Rate.-It has been generally assumed that the maximum pulse rate reached during exercise occurs at the end. In exercise lasting one minute Peabody and Sturgis (1922) nearly always found the maximum rate during the last 15 seconds. Essex et al. (1939), working on dogs, found that in an exercise lasting about 15 minutes the maximum rate for a given amount of work was usually reached within one to three minutes of the beginning of the exercise, and that frequently the pulse rate fell well below the initial rise and remained fairly constant at the lower level. They pointed out that these findings in the dog are in striking contrast with those of previous workers, who found that in man the pulse rate continues to increase throughout the period of exercise until the maximal rate is reached.

The present findings closely resemble those of Essex et al. The maximum rate, instead of occurring at the end of the 24 seconds of exercise, is reached on the average 15 seconds after exercise begins. After this maximum the rate falls slightly and continues more or less level till the end. This, which is well shown in Fig. 2, does appear in the records of other workers, though it has

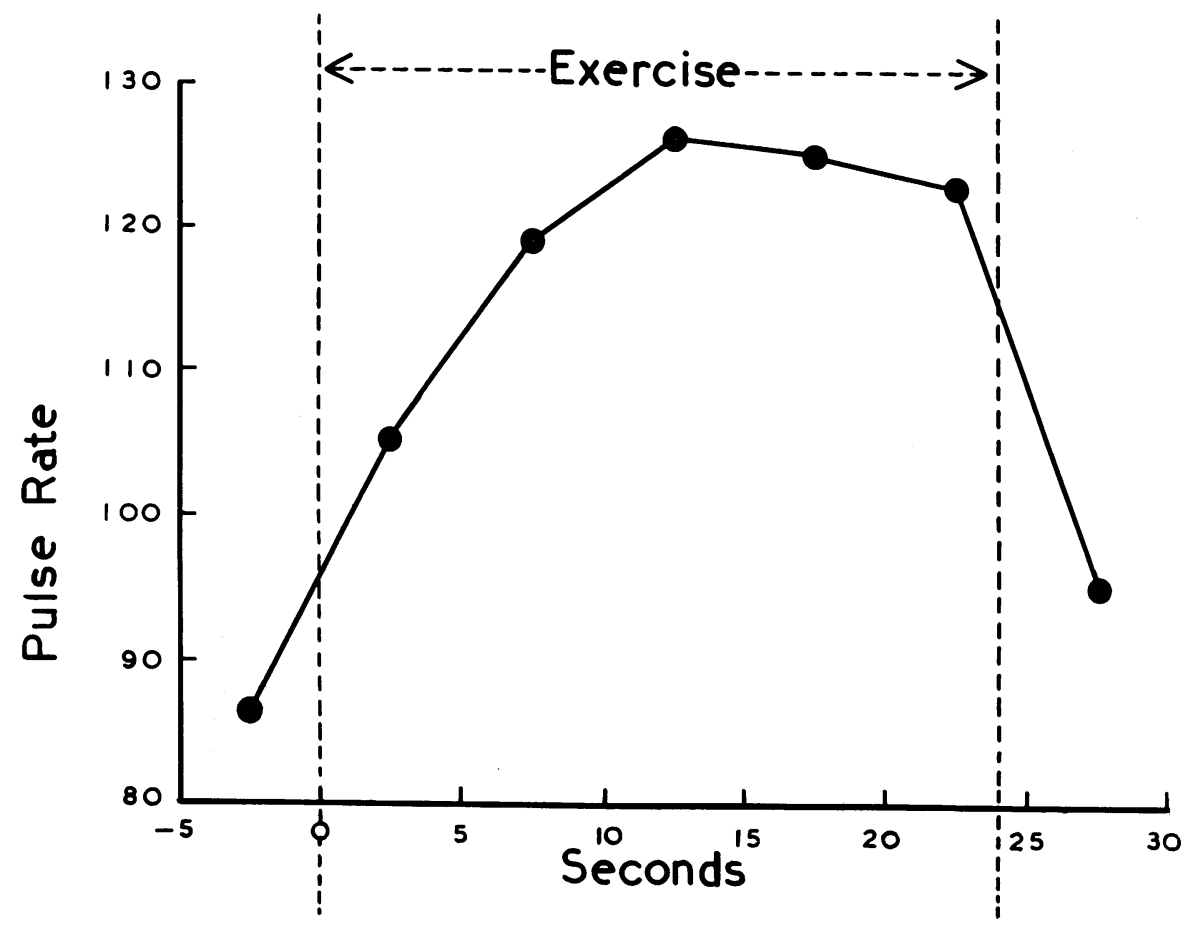

FIG. 2.-The average pulse rates of 75 male students in 5-seconds intervals before, during, and after the standard exercise. The average maximum rate here is slightly lower than that given in the table because the maximum rate does not always fall in the same 5-seconds interval.

not been stressed by them. In experiments on light work (tapping a key) by Bowen (1903) the average curve showed the maximum rate after two and a half minutes in an exercise lasting four minutes. Boas and Goldschmidt (1932) noted that in boys performing exhausting exercise for over three minutes the maximum rate was generally found two minutes after exercise began. 
Paterson (1928) found in some cases an early maximum rate followed by a decline before a subsequent slower rise to the level of the early maximum. A curve given by Gillespie, Gibson, and Murray (1925) for light work also showed this effect, and Backwell (1921) (quoted by McDowall, 1939), obtained the same result when the start of the effort was sudden.

It was thought that our early maximum and subsequent slowing during exercise might be because the exercise was started from the sitting posture. Standing up might augment the rate during the first half of the exercise and this might pass off as circulatory adjustments are made. This, however, is not the case, as trials made with exercise starting from the standing posture still show the early maximum rate.

There are two main possibilities concerning the behaviour of the heart when an increased demand is made on the circulation.

The first theory postulates that the amplitude of the heart beat, i.e. its stroke volume or output per beat, is variable; and thus the reserve capacity of the circulation lies mainly in variations of stroke volume and rate. The second theory postulates that the stroke volume remains nearly constant, and the demands on the circulation during activity are met mainly by variations in rate. A factor of safety is provided during exercise because the tissues are then able to take more oxygen out of a given volume of blood. This theory of the constant output per beat was supported by Henderson (1923 and 1925), while Henderson, Haggard, and Dolley (1927) found that the output per beat remained relatively constant during rest and moderate exertion in ordinary subjects, but that it might be much increased in athletes performing strenuous exercise.

The first theory, that of variable output per beat, was stated by Bainbridge (1919) and was supported by the experiments of Means and Newburgh (1915), and by later work of Lythgoe and Pereira (1925). On this basis, a theory to account for the early maximum rate and subsequent slowing of the heart during the exercise might be put forward as follows. Assuming the frequency and the output per beat to be independently variable, the increase in blood flow required by the exercise might be brought about in the early stages (the first fifteen seconds or so) mainly by increase in the rate, thus giving the rapid rise to the maximum rate. Then, in order to minimize strain on the heart muscle, the output per beat might increase enough to allow a slight slowing off in the rate, the exercise ending before any further increase in rate is needed. The observations of Means and Newburgh on the effects of increasing amounts of work tended to show that the output per beat increased before the frequency.

(4) Acceleration of the Heart Rate.-The chief point of interest of this index is that it is a measure of the acceleration of the heart rate, expressed as beats-per-minute per second, and not merely an expression of change of rate. It is, however, very variable, the coefficient of variation being as high as 28.7 per cent. As was pointed out long ago by Bowen (1903), Buchanan (1909), and others, the acceleration begins immediately the exercise starts, and this can clearly be seen on the tracings.

(5) Number of Extra Beats induced by the Exercise.-This index is of no great value in the present series of healthy men, but preliminary tests on patients 
with heart lesions suggest it may be of interest. There was no significant correlation of extra beats and body weight in the present series.

(6) Percentage Increase on Initial Rate.-This has a high coefficient of variation and the variations occur over a wide range, no doubt because the maximum rate remains relatively constant while the initial rate varies widely. Hill, Magee, and Major (1937) found the percentage increase of pulse rate after two maximum pulls on a dynamometer to be very variable, but the absolute rise in rate was about the same in those with slow, medium, or fast resting rates.

(7) Actual Increase in Beats per Minute caused by the Exercise.-The relationship of this to the initial rate is dealt with under " maximum rate." The absolute increase in beats per minute here is very variable, in contrast with the results obtained by Hill, Magee, and Major (1937).

(8) Heart Rate in the 30 Seconds beginning 5 Seconds after Exercise.-This is highly correlated with the maximum rate, the coefficient being +0.83 with a standard error of $0 \cdot 116$. This index, then, which requires no apparatus and can be obtained by palpation, is a fairly reliable guide to the maximum rate reached during the exercise. The regression equation is : maximum rate $=0.72$ post-exercise rate +64 .

Cotton and Dill (1935) using a modified Boas' cardiotachometer recorded the heart rate in four periods of ten seconds each, two immediately preceding and two immediately following the end of exercise. The exercise consisted o walking and running on a flat treadmill and was continued long enough for a steady state to be reached. They concluded that the heart rate during exercise may be predicted from that recorded in the ten seconds following exercise, with an error whose standard deviation is less than 3 per cent; and that the heart rate fell very little during the first ten seconds after the exercise, and for the next ten seconds only about 6 per cent. The conditions differed from those of the present series in that their exercise was prolonged until a steady state was reached; which, no doubt, accounts for the slow drop after the exercise, in contrast to the rapid drop observed in the present series (see Fig. 2). Gillespie, Gibson, and Murray (1925) stated that while the pulse rate just after exercise was a fairly reliable relative indication of the rate during the exercise, it was impossible to draw inferences therefrom as to the absolute rate.

Paterson (1928) found that the values of the pulse rate during exercise were much higher than one would expect from values taken even immediately after exercise. This is upheld by the present results.

\section{Sinus Arrhythmia}

Treadgold (1930) said that sinus arrhythmia was usually of no significance as regards cardiac efficiency unless it was extremely marked. As it is easily detected by the present method of recording, it may be of interest to give its frequency of occurrence. The tracings have been divided into four categories according to the degree of sinus arrhythmia. It was absent in 9 per cent; slight in 29 per cent; marked in 55 per cent; and very marked in 7 per cent. 
Thus it will be seen that the condition was present to a marked degree in over 60 per cent of these normal young adults. Fig. 3 shows one such case. In

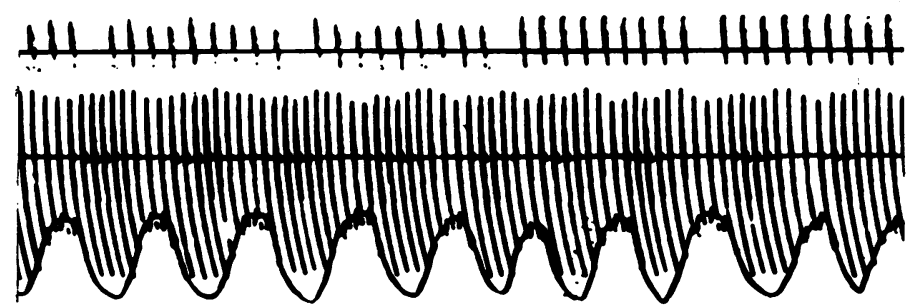

FIG. 3.-This tracing shows marked sinus arrhythmia. Upper tracing, time in seconds. Middle tracing, heart beats. Lower tracing, respiration (the rise of the lever indicates expiration, the fall indicates inspiration).

most cases the sinus arrhythmia disappeared during the exercise, but was especially prominent after it, perhaps due to deeper breathing. Dana (1919) described simple pauses in the heart beat, apparently dropped beats, which occurred in association with simple sinus arrhythmia. About 15 per cent of the present series show this condition in some degree (Fig. 4), but not always
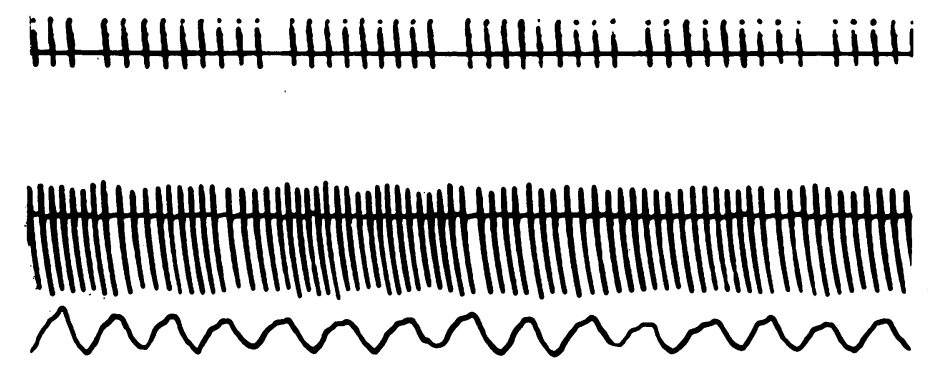

FIG. 4.-This tracing shows simple pauses in the rhythm of the heart. Upper tracing, time in seconds. Middle tracing, heart beats. Lower tracing, respiration.

those with marked sinus arrhythmia. The condition would appear to be some form of sinus block.

It is hoped that this somewhat exhaustive survey of results in a simple cardiac tolerance test will serve as a basis for further tests which are being carried out on the heart rate during exercise in patients with various cardiac esions.

\section{SUMmaRY}

A simple two-step cardiac tolerance test of very brief duration is described, in which the subject's heart beats are electrically recorded on a smoked drum throughout the test.

From the analysis of the records of 75 healthy male students, the following indices are obtained and discussed: initial rate; maximum rate; time to reach the maximum rate; acceleration of the heart rate; number of extra beats induced by the exercise; actual and percentage increase on initial rate; and the rate after exercise.

The mean values and variability of these indices are given. 
I wish to acknowledge my indebtedness to Professor E. P. Cathcart for his interest and encouragement during the progress of the work.

\section{REFERENCES}

Addis, T. (1922). Arch. intern. Med., 29, 539.

Backwell (1921). Private communication to Professor McDowall.

Bainbridge, F. A. (1919). The Physiology of Muscular Exercise, London, p. 53.

Bell, G. H., and Knox, J. A. C. (1938). J. Physiol., 93, 36P.

Bell, G. H., Knox, J. A. C., and Small, A. J. (1939). Brit. Heart Jour., 1, 229.

Boas, E. P., and Goldschmidt, E. F. (1932). The Heart Rate, London.

Bowen, W. P. (1903). Contributions to Medical Research dedicated to V. C. Vaughan, Michigan, p. 462.

Buchanan, F. (1909). Trans. Oxford Jun. Scientific Club, 34, 351.

Cotton, F. S., and Dill, D. B. (1935). Amer. J. Physiol., 111, 554.

Cotton, T. F., Rapport, D. L., and Lewis, T. (1917). Heart, 6, 269.

Dana, H. W. (1919). Amer. J. med. Sci., 157, 750.

Ellis, L. B. (1932). Amer. J. Physiol., 101, 494.

Essex, H. E., Herrick, J. F., Baldes, E. J., and Mann, F. C. (1939). Amer. J. Physiol., 125, 614.

Gillespie, R. D. (1924). J. Physiol., 58, 425.

Gillespie, R. D., Gibson, C. R., and Murray, D. S. (1925). Heart, $12,1$.

Grollman, A. (1929). Amer. J. Physiol., 89, 584.

Henderson, Y. (1923). Physiol. Rev., 3, 165. (1925). Lancet, 2, 1265 and 1317.

Henderson, Y., Haggard, H. W., and Dolley, F. S. (1927). Amer. J. Physiol., 82, 512.

Hill, A. B., Magee, H. E., and Major, E. (1937). Lancet, 2, 441.

Jackson, C. M. (1927). Amer. J. Anat., 40, 59.

Langowoy, A. P. (1900). Dtsch. Archiv. f. klin. Med., 68, 268.

Lythgoe, R. J., and Pereira, J. R. (1925). Proc. Roy. Soc. B, 98, 468

McCurdy, J. H., and Larson, L. A. (1939). The Physiology of Muscular Exercise, 3rd ed., London.

McDowall, R. J. S. (1939). The Control of the Circulation of the Blood, London, p. 413.

Master, A. M. (1934). Amer. Heart J., 10, 495.

Master, A. M., and Oppenheimer, E. T. (1929). Amer. J. med. Sci., 177, 223.

Means, J. H., and Newburgh, L. H. (1915). J. Pharmacol., 7, 441.

Paterson, W. D. (1928). J. Physiol., 66, 323.

Peabody, F. W., and Sturgis, C. C. (1922). Arch. intern. Med., 29, 277.

Treadgold, H. A. (1930). Proc. Roy. Soc. Med., 23, (2), War Section, p. 7.

Volkmann, A. W. (1850). Die Haemodynamik nach Versuchen, Leipzig, p. 427.

White, P. D. (1937). Heart Disease, 2nd ed., New York, p. 158. 